Article

\title{
Helicase-Like Transcription Factor HLTF and E3 Ubiquitin Ligase SHPRH Confer DNA Damage Tolerance through Direct Interactions with Proliferating Cell Nuclear Antigen (PCNA)
}

\author{
Mareike Seelinger and Marit Otterlei *(D) \\ Department of Clinical and Molecular Medicine, Faculty of Medicine and Health Sciences, \\ Norwegian University of Science and Technology NTNU, N-7491 Trondheim, Norway; \\ mareike.seelinger@ntnu.no \\ * Correspondence: marit.otterlei@ntnu.no; Tel.: +4792889422
}

Received: 12 December 2019; Accepted: 19 January 2020; Published: 21 January 2020

check for updates

\begin{abstract}
To prevent replication fork collapse and genome instability under replicative stress, DNA damage tolerance (DDT) mechanisms have evolved. The RAD5 homologs, HLTF (helicase-like transcription factor) and SHPRH (SNF2, histone-linker, PHD and RING finger domain-containing helicase), both ubiquitin ligases, are involved in several DDT mechanisms; DNA translesion synthesis (TLS), fork reversal/remodeling and template switch (TS). Here we show that these two human RAD5 homologs contain functional APIM PCNA interacting motifs. Our results show that both the role of HLTF in TLS in HLTF overexpressing cells, and nuclear localization of SHPRH, are dependent on interaction of HLTF and SHPRH with PCNA. Additionally, we detected multiple changes in the mutation spectra when APIM in overexpressed HLTF or SHPRH were mutated compared to overexpressed wild type proteins. In plasmids from cells overexpressing the APIM mutant version of HLTF, we observed a decrease in C to $\mathrm{T}$ transitions, the most common mutation caused by UV irradiation, and an increase in mutations on the transcribed strand. These results strongly suggest that direct binding of HLTF and SHPRH to PCNA is vital for their function in DDT.
\end{abstract}

Keywords: Translesion synthesis (TLS); mutagenicity; DNA damage tolerance (DDT); APIM; RAD5

\section{Introduction}

Cells are constantly exposed to endogenous and exogenous agents that cause DNA lesions. DNA translesion synthesis (TLS), fork reversal, and template switch (TS) are DNA damage tolerance (DDT) mechanisms handling DNA lesions or other obstacles during replication. This is achieved by one or more specialized low-fidelity polymerases in TLS (TLS polymerases), by converting the replication fork into a stabilized chicken-foot structure intermediate, or by using the nascent strand in the sister chromatid as a template in TS. These mechanisms allow cells to continue replication and to prevent replication fork collapse [1].

One day of sun exposure is estimated to induce up to $10^{5} \mathrm{UV}$-induced photolesions per cell [2]. UVB irradiation is absorbed by the DNA and induces mainly two types of UV-photodimers: cyclobutane pyrimidine dimers (CPDs) and pyrimidine pyrimidone (6-4)photoproducts (6-4PPs). CPDs account for the largest fraction of all photoproducts [3,4]. They are the major mutagenic UV lesion in mammalian cells, because they exhibit a slower repair-rate and are therefore often bypassed by TLS polymerases [5-7]. However, 6-4PPs, on the other hand, are bulky lesions which are rapidly detected and repaired by nucleotide excision repair (NER) [8]. The Xeroderma Pigmentosum variant (XPV)

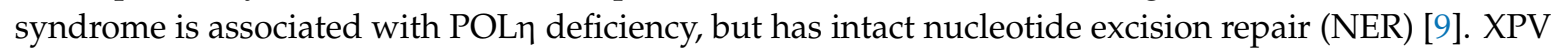


patients are hypersensitive toward sunlight and have an $~ 1000$ times higher skin cancer incidence rate [10-12], illustrating the importance of properly regulated TLS, even in presence of functional NER (reviewed in [13]).

The DDT mechanisms are at least partly coordinated by mono- and polyubiquitination of K164 of PCNA (reviewed in [14]). The RAD5 homologues, HLTF and SHPRH, are ubiquitin ligases containing a RING domain which is involved in polyubiquitination of PCNA [15]. They are therefore believed to be important for inducing TS. In addition, HLTF mediates fork reversal via its HIRAN domain interacting with the 3'ends of a frayed fork [16]. For SHPRH, which lacks a HIRAN domain, fork reversal has not been reported yet. Human cells have at least two additional important fork reversal proteins, SMARCAL1 and ZRANB3. The latter contains a functional PCNA interacting sequence, AlkB homolog 2 PCNA interacting motif (APIM) [17]. In addition, SMARCAL1, ZRANB3, and HLTF can restore a three-way-replication fork from a four-way-reversed replication fork [16,18]. Furthermore,

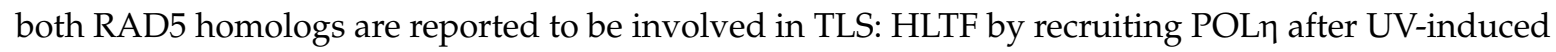
DNA damage, and SHPRH by recruiting POLk after methyl methanesulfonate (MMS)-induced DNA damage [19]. HLTF and SHPRH are regarded as candidate tumor suppressor genes, because loss of function or dysregulation has been linked to cancer development [20-22], and HLTF is often epigenetically silenced by promotor hypermethylation in colon cancers $(\sim 40 \%)$ [23]. Interestingly, both RAD5 homologs contain potential PCNA interacting motifs, APIMs, within the helicase domain at their C-terminus; KFIVK (amino acid (aa) 959-963) in HLTF and RFLIK (aa 1631-1635) in SHPRH.

Serving as a scaffold protein, PCNA switches between protein interaction partners, using either of the two motifs: the PIP-box or APIM. While the majority of APIM-containing proteins are involved in various cellular stress responses, including DNA repair, TS and TLS, the PIP-box is found in multiple proteins essential for replication [24-26]. The two motifs share the same interaction site on PCNA, which is a potential binding site for over 600 proteins [27-30]. Interactions with PCNA are therefore coordinated at multiple levels, including affinity-driven competitive inhibition, context/cellular state and posttranslational modifications on either PCNA or the interacting proteins [17,31]. Data suggest that cellular stress, e.g., such as replication stress, is important for increased APIM-PCNA affinity $[17,24,26,27,32-34]$. Indeed, functional APIM is verified in three proteins directly involved in DDT, ZRANB3 [17], TFII-I [24,35], and the catalytic subunit of POL $\zeta$, REV3L [36], and in two proteins involved in the regulation of TS, FBH1 [28], and RAD51B [24]. Here we show that APIM in both HLTF and SHPRH interact with PCNA, and that the binding of HLTF and SHPRH to PCNA is important for their function in DDT after UV-induced DNA damage.

\section{Results and Discussion}

\subsection{APIM in HLTF Is a Functional PCNA Interacting Motif}

APIM in HLTF (KFIVK) fused to CFP and co-expressed with HcRed-tagged PCNA colocalized with PCNA in foci resembling replication foci (Figure 1A, mid two images). We have previously shown that a mutation of the second position in APIM from an aromatic amino acid to alanine reduced the affinity to PCNA by 50\% [37]. Accordingly, the HLTF APIM F2A mutant (KAIVK) fused to YFP did not colocalize with PCNA in foci when co-expressed with KFIVK-CFP and HcRed-PCNA (Figure 1A, left image), a first indication for the importance of APIM in HLTF. However, both wild type and APIM mutant versions (F960A) of full-length YFP-HLTF colocalized with overexpressed HcRed-PCNA (Figure 1D,H). The persistent colocalization of YFP-HLTF F960A with PCNA in replication foci indicates that the mutation in APIM only reduces, but does not abolish the interaction with PCNA, and that other proteins partners participating in the same complex may interact with PCNA. The cellular localization of YFP-HLTF F960A was, however, slightly different from the wild type protein, i.e., we detected a higher level of fluorescence in the cytosol. Thus, the mutation in APIM of HLTF might have reduced the stability, reduced nuclear retention, or increased nuclear export of the protein (Figure 1B, quantified in C). Therefore, we next tested if the nuclear level of HLTF is actively regulated. The nuclear HLTF level 
was measured after overexpression and treatment of the cells with Leptomycin B (an active nuclear export blocking drug). We found increased nuclear levels of both YFP-HLTF and YFP-HLTF F960A (Figure 1C). This indicates that the level of HLTF in the nucleus is actively regulated, but that the export of HLTF is independent of APIM and thus independent of a direct HLTF-PCNA interaction (Figure 1C).

To further investigate the importance of APIM in HLTF for colocalization with PCNA in replication foci, HLTF and HLTF F960A were overexpressed together with APIM-peptides and PCNA. The intensity of YFP-HLTF in PCNA foci was significantly reduced by co-expression of KFIVK-CFP (APIM in HLTF), and an even stronger reduction could be achieved by overexpression of RWLVK-CFP, an APIM-version with increased PCNA-affinity [36] (Figure 1E,F, quantified in G). The intensity of YFP-HLTF F960A in PCNA foci was initially stronger than YFP-HLTF; however, after overexpression of APIM-peptides (KFIVK-CFP or RWLVK-CFP), foci intensity was reduced to the same or lower level as measured for YFP-HLTF (Figure 1I,J, quantified in K). These results show that localization of both wild type HLTF and HLTF F960A in PCNA foci are reduced by overexpression of peptides containing the APIM sequence of HLTF, supporting that APIM in HLTF is a functional PCNA interacting motif. 

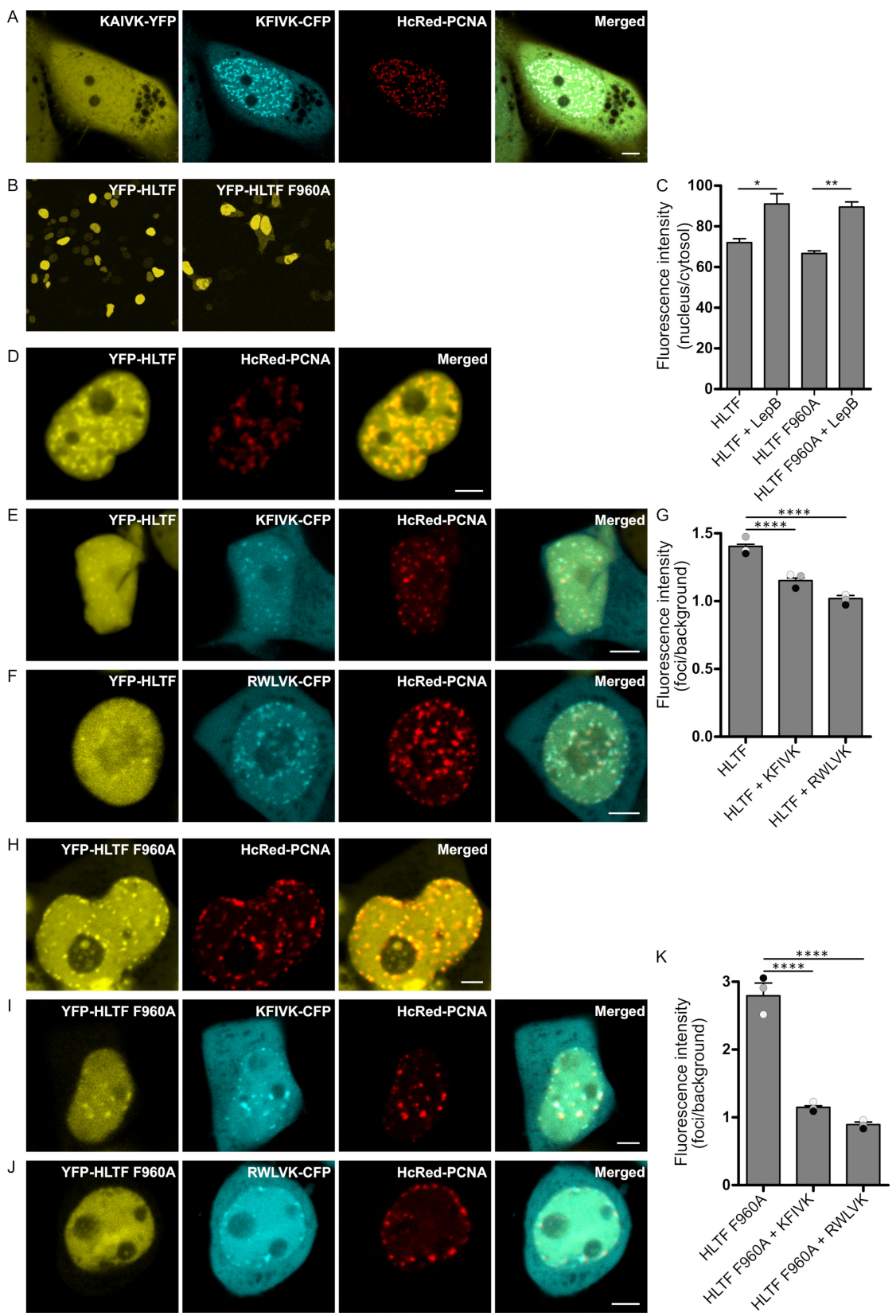

Figure 1. APIM in HLTF is a functional PCNA interacting motif. (A) Overexpressed KAIVK-YFP (mutAPIM in HLTF), KFIVK-CFP (APIM in HLTF), and HcRed-PCNA. (B) Overview of subcellular localization of YFP-HLTF and YFP-HLTF F960A, and (C) quantification of their nuclear localization in cells treated with Leptomycin B (LepB; $30 \mathrm{ng} / \mathrm{mL}, 45 \mathrm{~min})(n=>90$ cells per sample). (D) Overexpressed YFP-HLTF and HcRed-PCNA; (E) YFP-HLTF, KFIVK-CFP (APIM of HLTF) and HcRed-PCNA; and (F) YFP-HLTF, RWLVK-CFP, and HcRed-PCNA. (G) Quantification of YFP-HLTF foci intensities alone $(n$ (number of foci) $=132)$ and with co-transfection of KFIVK-CFP $(n=76)$ or RWLVK-CFP ( $n=59$ ) from three biological replica depicted in white, gray, and black dots. Bars represent averages. (H) Overexpressed YFP-HLTF F960A and HcRed-PCNA; (I) YFP-HLTF F960A, KFIVK-CFP (APIM of HLTF), and HcRed-PCNA; and (J) YFP-HLTF F960A, RWLVK-CFP, and HcRed-PCNA. (K) Quantification of YFP-HLTF F960A foci intensities alone $(n=84)$ and with co-transfection of KFIVK-CFP $(n=103)$ or RWLVK-CFP $(n=98)$ from three biological replica depicted in white, gray, and black dots. Bars represent averages. Quantifications in $\mathrm{G}$ and $\mathrm{K}$ are based on at least 10 different images per confocal dish/sample in cells with similar protein expression. Foci intensity quantifications were done by using processing software Fiji (ImageJ). Two-sided Student's $t$-test, ${ }^{*} p<0.05,{ }^{* *} p<0.01$, **** $p<0.0001$. All images are from live cells. Scale bar $=5 \mu \mathrm{m}$. 


\subsection{Nuclear Localization of SHPRH Depends on Its Interaction with PCNA}

Like HLTF, APIM in SHPRH (RFLIK) was fused to CFP and co-expressed with HcRed-tagged PCNA. RFLIK-CFP colocalized with PCNA, while the F2A APIM mutant version (RALIK-CFP) did not (Figure 2A). The same APIM mutation in full-length SHPRH (F1632A), led to a strong reduction in nuclear localization compared to wild type SHPRH (Figure 2B, quantified in C). These results could suggest that the interaction with PCNA is necessary for nuclear localization of SHPRH or that the mutant SHPRH protein is less stable. To explore if the nuclear localization of SHPRH is dependent on a direct interaction with PCNA, we examined if the fraction of nuclear SHPRH can be reduced by treatment with an APIM containing cell penetrating peptide (APIM-peptide), which has earlier been shown to block the binding of APIM-containing proteins to PCNA [27]. Indeed, the fluorescence intensity of GFP-SHPRH in the nucleus was reduced upon APIM-peptide treatment (Figure 2C), and this effect was not achieved by treatment with a mutant version of the APIM-peptide with reduced affinity for PCNA (MutAPIM-peptide, W2A) [37]. Together, these results indicate that the nuclear localization of SHPRH is dependent on its direct binding to PCNA via APIM.

Like HLTF, both GFP-SHPRH and GFP-SHPRH F1632A colocalized with overexpressed HcRed-PCNA in replication foci (Figure 2D,E). Thus, the residual affinity of SHPRH F1632A to PCNA and/or its interaction with other PCNA interacting proteins is sufficient to cause the observed localization of SHPRH F1632A in replication foci.

\subsection{APIM in SHPRH and HLTF Is Required for Maximal Pull Down of PCNA After DNA Damage}

Our results suggest that APIM in both HLTF and SHPTH are functional PCNA-interacting motifs. To further verify this, we examined if endogenous PCNA can be pulled down by the GFP-SHPRH and YFP-HLTF fusion proteins. As APIM-PCNA interactions are relatively weak, and PCNA-interactions with HLTF and SHPRH are expected to be dynamic and to increase upon DNA damage, we treated the cells with the DNA alkylating agent MMS and gently cross-linked the cells with formaldehyde before making extracts (see Material and Methods and [24]). We could not detect any specific PCNA pull down in absence of MMS treatment (data not shown), but both YFP-HLTF and GFP-SHPRH pulled down more endogenous PCNA than the YFP control (Figure 2F, normalized against total GFP pulled down). The mutated APIM versions of the proteins, especially SHPRH F1632A, pulled down less PCNA compared to the wild type proteins. We detected less full-length protein and more degraded protein of the APIM mutant versions compared to the wild type proteins (Supplementary Figure S1), particularly of HLTF F960A, and this may possibly also influence the level of PCNA pull down. However, increased degradation of the APIM mutant versions of HLTF and SHPRH may also support that their interaction with PCNA is important for the stability of these proteins. 

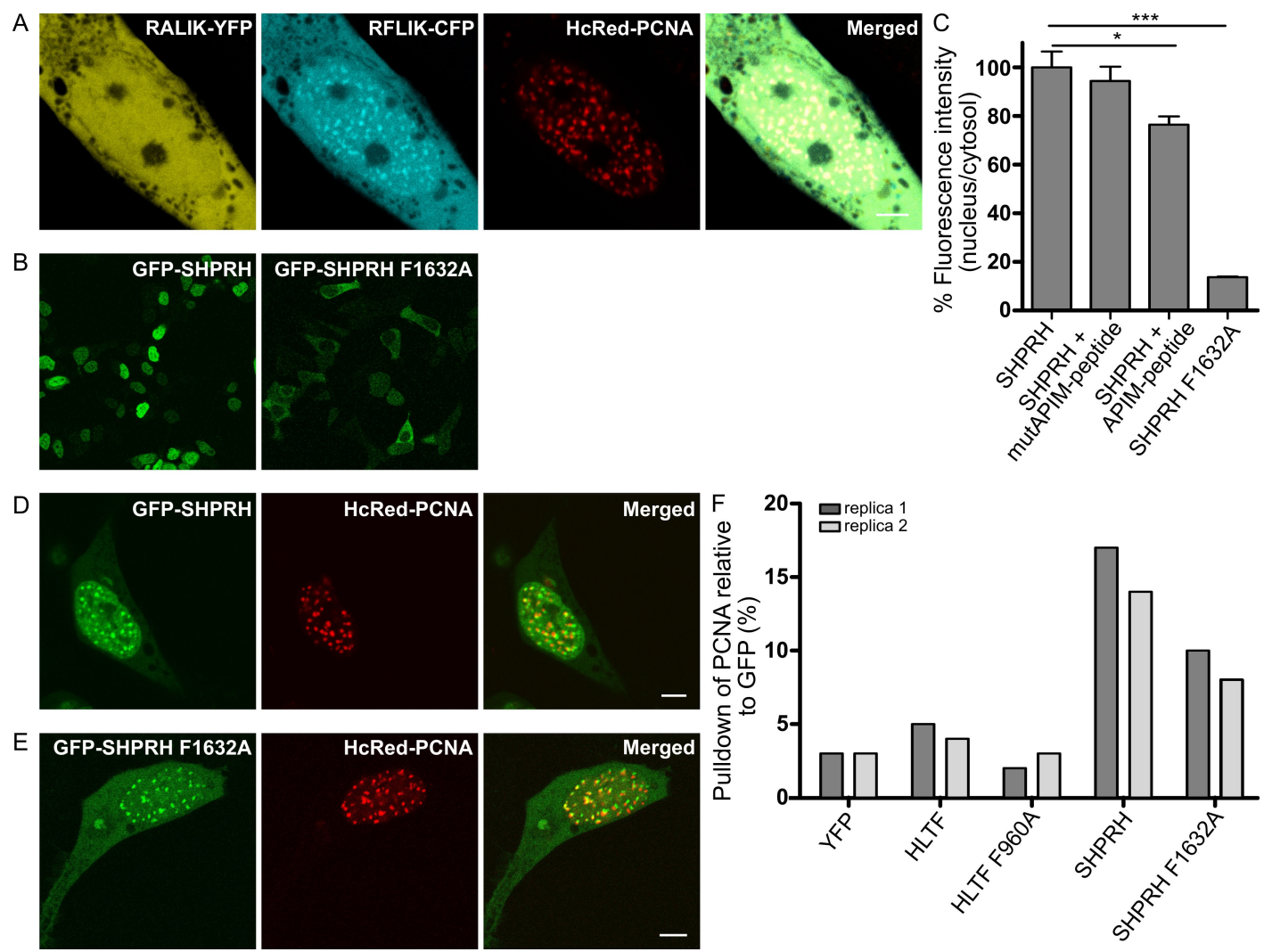

Figure 2. SHPRH localization in the nucleus is dependent on APIM. (A) Overexpressed RALIK-YFP (mutAPIM in SHPRH), RFLIK-CFP (APIM in SHPRH), and HcRed-PCNA. (B) Overview of subcellular localization of GFP-SHPRH and GFP-SHPRH F1632A, and (C) Quantification of nuclear localization of GFP-SHPRH ( $n$ (number of foci) $=484$ ) and GFP-SHPRH F1632A $(n=123)$, and GFP-SHPRH after treatment with an APIM peptide $(n=247)$ or mutAPIM-peptide $(n=319)$, average of three biological replica, normalized to untreated control, two-sided Student's $t$-test, ${ }^{*} p<0.05,{ }^{* * *} p<0.001$. (D) Overexpressed GFP-SHPRH and HcRed-PCNA and (E) GFP-SHPRH F1632A and HcRed-PCNA. All images are from live cells. Scale bar $=5 \mu \mathrm{m}$. (F) Quantification of PCNA level pulled down by anti-GFP from YFP-HLTF, YFP-HLTF F960A, GFP-SHPRH, and GFP-SHPRH F1632A transfected cells after weak cross-linking and MMS treatment. Level of PCNA is given as \% of total GFP-protein pulled down. Two independent biological replicas are shown.

\subsection{Direct Interaction with PCNA Is Important for the Regulation of DDT by HLTF and SHPRH}

To further examine the functionality and the impact of APIM in HLTF and SHPRH in DDT, we performed SupF mutagenesis assays. We measured the mutation frequency and analyzed the mutation spectra in UV-damaged reporter plasmids in cells overexpressing wild type or APIM mutant versions of HLTF or SHPRH. Overexpression of HLTF did not significantly change the mutation frequency compared to control, although a tendency toward a reduction was observed. However, overexpression of HLTF F960A significantly increased the mutation frequency by $45 \%$, compared to both control and HLTF wild type overexpressing cells (Figure 3A). This suggests that a direct interaction with PCNA is important for HLTF's ability to support error-free repair. One of the most

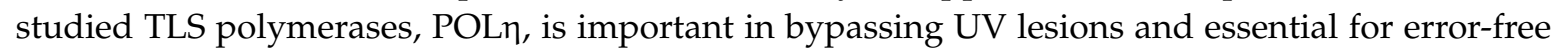
bypass of TT-CPDs [38]. HLTF has previously been reported to stimulate bypass by POLn [19].

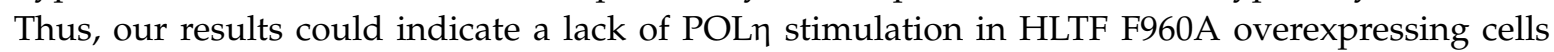
and that POLn stimulation by HLTF is dependent on a direct interaction with PCNA. Recently, a loss-of-function mutation in HLTF has been reported to increase the amount of DNA damage due to a decrease in PCNA polyubiquitination [39]. Thus, the increase in mutation frequency in presence of HLTF F960A overexpression could also be caused by a reduction of PCNA polyubiquitination 
and TS, indicating that APIM in HLTF is important for PCNA polyubiquitination mediated by HLTF. Recently, Masuda and colleagues reported that a HLTF-PCNA interaction at stalled primer ends reduced the polyubiquitination activity of HLTF in vitro, and that the polyubiquitination activity of HLTF could be partly restored by a mutation in the putative APIM in HLTF (F960A) [40]. However, they did not know if APIM was a functional PCNA interacting motif. Here we show that APIM in HLTF is a functional PCNA interacting motif; thus, our results support that the restoration of HLTF's polyubiquitination activity, seen by Masuda and colleagues, was due to a reduced PCNA interaction. Therefore, the increased mutation frequency in HLTF F960A overexpressing cells detected in our study is probably not caused by reduced, but rather by increased polyubiquitination and excessive fork reversal. The latter is associated with genomic instability [41].

SHPRH overexpression increased the mutation frequency compared to both SHPRH F1632A overexpressing and control cells (Figure 3A), suggesting dysregulated DDT after SHPRH overexpression.

In line with our data, it is reported that HLTF is more important for the regulation toward error-free DDT over UV-induced DNA damage than SHPRH, i.e., the mutation frequency was increased after HLTF knockdown but not after SHPRH knockdown [19]. The mutation spectra of supF reveal multiple differences between plasmids replicated in cells overexpressing wild type and APIM mutant versions of the RAD5 homologs. This further illustrates the impact of a direct interaction with PCNA for both HLTF and SHPRH (Figure 3B,C). 


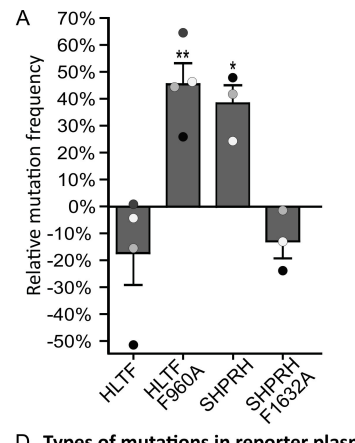

B \% Mutations at certain position C

C \% Mutations at certain position
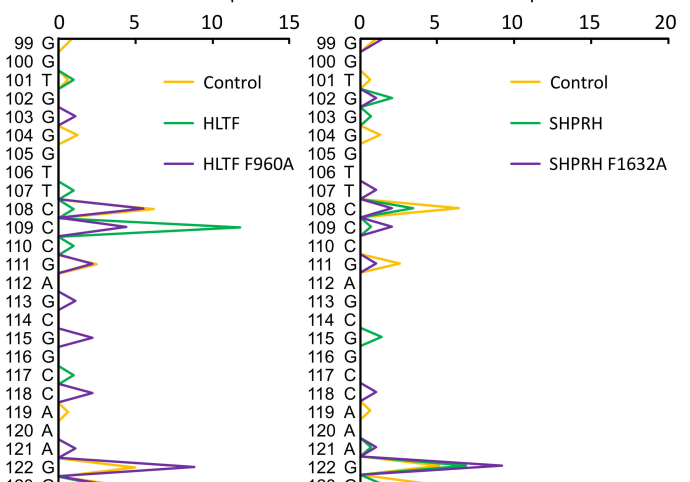

D Types of mutations in reporter plasmid (\%)


$\begin{array}{lll}124 \mathrm{G} & \\ 125 \mathrm{~A} & \\ 126 & \mathrm{G}\end{array}$

$\begin{array}{ll}126 & \mathrm{G} \\ 127 & \mathrm{C} \\ 128 & \mathrm{~A}\end{array}$

$128 \mathrm{~A}$

$\begin{array}{ll}130 & \mathrm{~A} \\ 131 & \mathrm{C} \\ 132 & \mathrm{~T}\end{array}$

$131 \mathrm{C}$
$132 \mathrm{~T}$
133
134
$135 \mathrm{~A}$
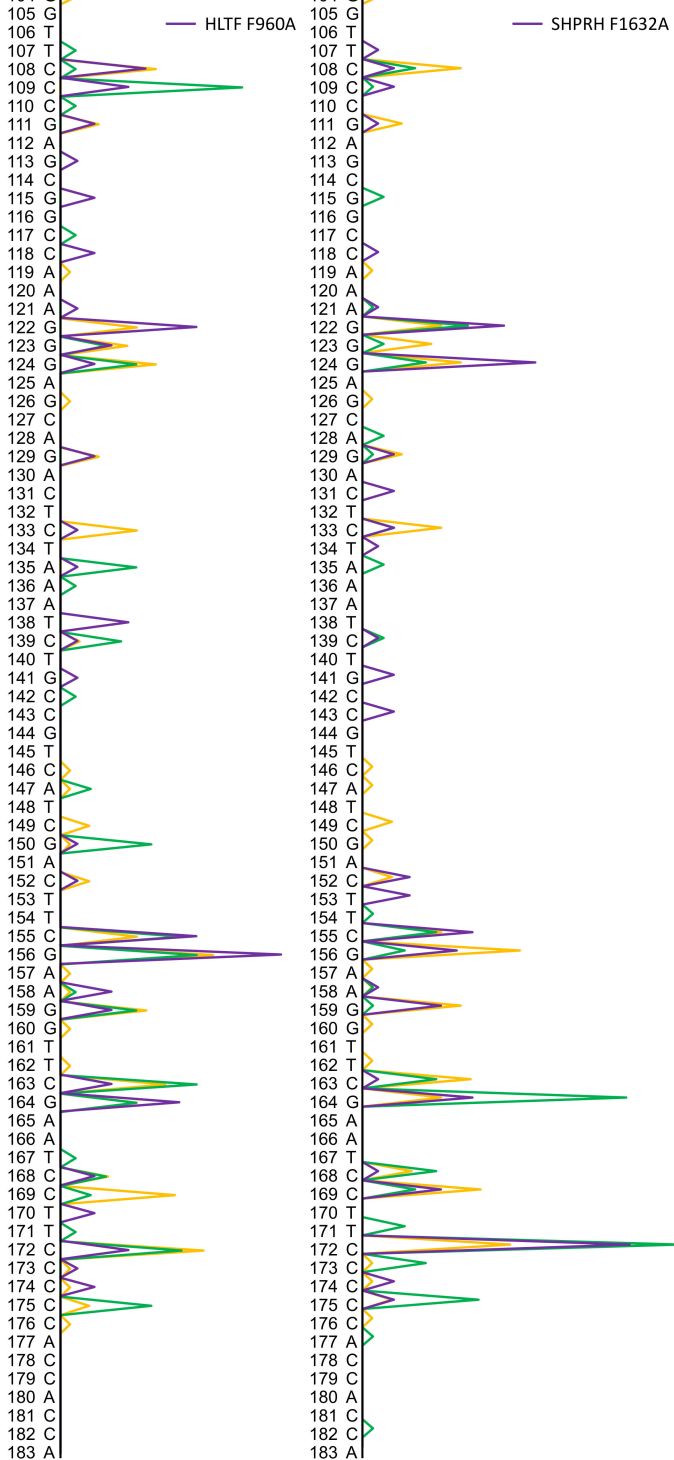

G



$183 \mathrm{~A}$

182 C

Figure 3. Mutation of APIM in HLTF and SHPRH results in differences in mutation frequencies and mutation spectra. (A) Mutation frequencies after overexpression of HLTF, HLTF F960A, SHPRH, or SHPRH F1632A, together with SupF reporter plasmid pSP189, relative to control (reporter plasmid only) in HEK293 cells from three or four biological replica (depicted as white, grey, and black dots). DNA from each biological replica was transformed until obtaining 1000-4000 colonies per replica. The total number of colonies counted are: HLTF $(n=14700)$, HLTF F960A $(n=13321)$, SHPRH $(n=11183)$, SHPRH F1632A ( $n=14709)$, and control $(n=17560)$. Two-sided Student's $t$-test relative to control, ${ }^{*} p<0.05,{ }^{* *} p<0.01$. $(B, C)$ Mutation spectra received from sequencing mutant colonies from (A). HLTF ( $n=102)$, HLTF F960A ( $n=91)$, SHPRH $(n=152)$, SHPRH F1632A $(n=102)$, and control $(n=163)$. (D) Quantification of different mutations using sequencing results from $(B, C)$. Mutations with prevalence $\geq 2 \%$ are shown. Mutations at $\mathrm{T}$ or $\mathrm{C}$ bases (putative coding strand mutations) in supF (E) Mutations at position 164 and 172 in supF isolated from cells overexpressing HLTF, HLTF F960A, SHPRH, or SHPRH F1632A compared to control. (F) Mutations at position 109 and 122 in supF isolated from cells overexpressing HLTF, HLTF F960A, SHPRH, or SHPRH F1632A compared to control. (G) Distribution of mutations using sequencing results from $(B, C)$ received from reporter plasmids isolated from control cells. 


\subsection{Direct Binding of HLTF to PCNA Is Important for Error-Free DDT and/or DNA Repair}

When analyzing the mutations in supF isolated from the different cells, we found mainly $\mathrm{C}$ to $\mathrm{T}$ transitions (corresponding $\mathrm{G}$ to A mutations on the transcribed strand) in plasmids from control cells (Figure 3D,G). DNA mutations resulting from UV exposure are usually $\sim 80 \% \mathrm{C}$ to $\mathrm{T}$ transitions [42], and, accordingly, $\mathrm{C}$ to T mutations are frequently found in skin cancers [43]. Thus, our results follow the expected mutation pattern from UV irradiation in vitro and in vivo. Overexpression of HLTF led to an increase of transitions from $83 \%$ (in control) to $90 \%$, and this increase was mainly caused by an increase in tandem CC to TT mutations (Figure 3D). UV induced 6-4PPs are quickly repaired by NER, while CPDs are repaired at a slower rate. Therefore, the main amount of mutations received in our experiments likely arose from the bypass of CPDs (TT, TC, and CC). C to T transitions are the

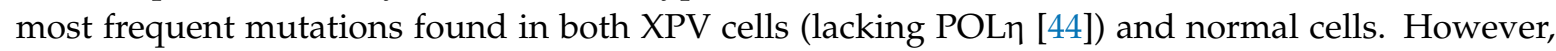

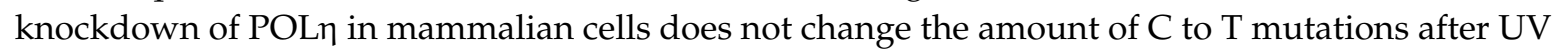

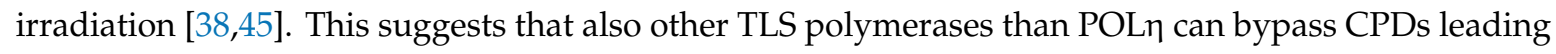
to $C$ to $\mathrm{T}$ transitions. Thus, whether the increase in CC to TT mutations is a result of HLTF mediated stimulation of POL $\eta$ or stimulation of other TLS polymerases cannot be concluded based on our data.

Overexpression of HLTF F960A resulted in fewer transition mutations compared to HLTF overexpression, especially on the coding strand (26\% versus $42 \% \mathrm{C}$ to $\mathrm{T}$ mutations, and $7 \%$ versus $12 \%$ CC to TT mutations), and an overall increase in mutations on the transcribed/noncoding/template strand (Figure 3D). At the same time, HLTF F960A overexpression led to an increase in mutation frequency (Figure 3A). Since transcription-coupled NER is error-free and probably repairs part of the lesions on the transcribed strand, HLTF F960A overexpression is suggested to either dysregulate NER and/or other error-free repair/bypass processes, for example, fork reversal or TS. Thus, our results suggest that a direct interaction between PCNA and APIM in HLTF is important for error-free TLS and/or DNA repair regulated by HLTF.

\subsection{SHPRH Overexpression Stimulates Error-Prone TLS}

Overexpression of SHPRH led to an increase of mutation frequency by $38 \%$ (Figure $3 \mathrm{~A}$ ) and an increase in transitions, mainly due to a higher amount of $\mathrm{C}$ to $\mathrm{T}$ transitions (51\% compared to $43 \%$ in control) (Figure 3D). Mutations at position 164 and 172 further illustrate this higher amount of mutations at Cs in SHPRH overexpressing cells (Figure 3E). POL $k$ has been shown to be activated by SHPRH overexpression [19], and thus the observed C to T transitions could be mediated by POLk. In the presence of SHPRH F1632A overexpression, we observed a mutation spectrum that was more similar to the control than to SHPRH wild type overexpression. This might be caused by lower nuclear level of SHPRH F1632A, because function and nuclear localization of SHPRH was dependent on a direct APIM-mediated PCNA interaction (Figure 3B).

Since HLTF and SHPRH are reported to be partly competitive [19,38], the changes in mutation patterns observed after overexpression of both wild type and APIM mutants of SHPRH and HLTF result from a disturbance of the HLTF/SHPRH ratio.

\subsection{Reduced Level of Putative Transcribed Strand Mutations after Overexpression of HLTF and SHRPH}

Due to the nature of UV lesions (mainly occurring at $C$ and $T$ bases) and the fact that we sequenced the coding strand only, we categorized mutations into mutations which with high probability occurred on the coding strand versus transcribed strand. We found a lower fraction of mutations on the coding strand ( $44 \%$ vs. $56 \%$ on transcribed strand) in supF isolated from control cells (Figure 3D). This could be explained by transcription coupled NER repairing lesions on the transcribed strand and/or by a higher TLS rate on the coding strand. When HLTF or SHPRH were overexpressed, the mutations on the coding strand decreased to $40 \%$. This could be explained by increased TLS on the coding strand (e.g., POL $\eta$ or k) or decreased error-free repair/bypass on the transcribed strand (e.g., TS, NER or error-free TLS). Overexpression of HLTF F960A resulted in an increased amount of G to A (48\%) transitions compared 
to overexpression of wild type HLTF (29\%) (Figure 3D). In addition, mutations on the transcribed strand increased from $40 \%$ for HLTF to $55 \%$ for HLTF F960A. The number of mutations at position 109 (mutations on coding strand CC) and 122 (mutations on transcribed strand CC) in the supF gene (Figure 3F) further illustrates the large difference between HLTF and HLTF F960A overexpression on coding and transcribed strand mutations. The same trends, although less pronounced, were detected for SHPRH wild type versus SHPRH F1632A, in spite of the low nuclear concentration of the mutated proteins. SHPRH overexpression resulted in a shift from $\mathrm{G}$ to $\mathrm{A}(30 \%)$ mutations to $\mathrm{C}$ to $\mathrm{T}(51 \%)$ mutations in comparison to SHPRH F1632A overexpression (37\% and 46\%, respectively) (Figure 3D), and differences in the mutation spectra especially in the amount of mutations at positions 124 and 164 and in the type of mutation, e.g., at position 122 (Figure 3B,E). In total, these results further support that APIM in HLTF as well as in SHPRH are functional PCNA interacting motifs, which seem to be especially important for reducing mutations on the transcribed strand.

In conclusion, the results presented here show that both mammalian RAD5 homologs, HLTF and SHPRH, have functional APIM sequences and that their direct interactions with PCNA are important for the regulation of the DDT pathways. Our data also show that increased levels of wild type HLTF and SHPRH disturb the balance of error-free and error-prone DDT pathways, suggesting that their expression levels are normally strictly regulated.

\section{Material and Methods}

\subsection{Expression Constructs}

pEGFP-C2-HLTF and pEGFP-C2-SHPRH, the pSP189 reporter plasmid and E. coli strain MBM7070 described in [19], were kind gifts from Professor Karlene Cimprich, Department of Chemical and Systems Biology, Stanford University, USA. HLTF was sub-cloned into pEYFP-C1 vector (YFP-HLTF) and site-specific mutations at position F960A in HLTF, and F1632A in SHPRH were generated as described in [24]. The wild type APIM sequences from HLTF (aa 959-963, KFIVK) and SHPRH (aa 1631-1635, RFLIK), as well as the corresponding APIM mutant (F2A), were cloned as fusions with CFP or YFP, respectively, by using PECFP-N1 and pEYFP-N1 vectors with mutated ATG, similarly to RWLVK-CFP [24]. CFP-PCNA and HcRed-PCNA have previously been described [46].

\subsection{Cell Lines}

HEK293 and HEK293T (ATCC: CRL-1573, CRL 11268, respectively) were cultured in D-MEM ( $4.5 \mathrm{~g} / \mathrm{L}$ glucose; Sigma-Aldrich, Saint Louis, MO, USA). Media were supplemented with $10 \%$ fetal bovine serum (FBS; Sigma-Aldrich), $2.5 \mu \mathrm{g} / \mathrm{mL}$ of Fungizone ${ }^{\circledR}$ Amphotericin B (Gibco, Thermo Fischer Scientific, Waltham, MA, USA), $1 \mathrm{mM}$ of L-Glutamine (Sigma-Aldrich), and antibiotic mixture containing $100 \mu \mathrm{g} / \mathrm{mL}$ of penicillin and $100 \mu \mathrm{g} / \mathrm{mL}$ of streptomycin (Gibco). The cells were cultured at $37^{\circ} \mathrm{C}$ in a $5 \% \mathrm{CO}_{2}$ humidified atmosphere.

\subsection{SupF Assay}

The SupF mutagenicity assays were performed, essentially, as previously reported [19]. Briefly, the reporter plasmid pSP189 was irradiated with $600 \mathrm{~mJ} / \mathrm{cm}^{2}$ UVB $(312 \mathrm{~nm})$, with UV lamp Vilber Lourmat, Bio Spectra V5. HEK293 cells were transfected with constructs of interest and UVB- irradiated pSP189 (including plasmids not exposed to UVR as controls), using X-treme GENE HP transfection reagent according to manufacturer protocol (Roche diagnostics); at least 3 biological replicas were conducted ( 3 transfections). Cells were harvested after $48 \mathrm{~h}$ for both isolation of plasmid and Western analysis. Isolated plasmids were DpnI (NEB, Ipswich, MA, USA) restriction digested to exclude original bacterial plasmids, in order to continue with only replicated plasmids. Isolated plasmids were transformed into E. coli MBM7070 cells and plated on indicator X-gal/IPTG/Amp agar plates. Blue/White screening was performed, and mutation frequency (white/blue colonies) was calculated for the different samples for several transfections (at least 3 replica). White and light-blue colonies were 
picked for re-streaking and DNA sequencing of supF gene. Colonies that did not show a mutation in the sequencing results were afterward excluded, and the mutation frequency was recalculated.

\subsection{Imaging}

Live cell imaging of HEK293 transfected with pGFP-SHPRH, pGFP-SHPRH F1632A, pYFP-HLTF, pYFP-HLTF F960A, pHcRed-PCNA, and YFP/CFP constructs of the APIM/mutated APIM in HLTF or SHPRH were performed $24 \mathrm{~h}$ after transfection, using a Zeiss LSM 510 Meta laser scanning microscope equipped with a Plan-Apochromate $63 \times / 1.4$ oil immersion objective. GFP and CFP were excited/detected at $\lambda=458 \mathrm{~nm} / \mathrm{BP} 470-500 \mathrm{~nm}$. YFP was excited/detected at $\lambda=514 \mathrm{~nm} / \mathrm{BP} 530-600 \mathrm{~nm}$, and HcRed was excited/detected at $\lambda=543 \mathrm{~nm} / \mathrm{LP} 615 \mathrm{~nm}$, using consecutive scans. The thickness of the scanned optical slices was $1 \mu \mathrm{m}$.

Live cell imaging of HEK293T transfected with pYFP-HLTF or pYFP-HLTF F960A together with pKFIVK-CFP or pRWLVK-CFP and pHcRed-PCNA was performed as described above, but YFP was detected at BP535-590 $\mathrm{nm}$ in these experiments. Fluorescence intensities were measured in cells with comparable expression levels of YFP and CFP, using the imaging processing software Fiji (ImageJ) version 1.06.2016. Average intensity within an area of interest (foci) was measured and divided by average intensity in the nucleus outside foci.

For SupF assay transfection control, HEK293 cells were transfected with proportional amounts of transfection mix as used in the SupF assay. Live cell imaging was performed $48 \mathrm{~h}$ after transfection using Zeiss LSM 510 Meta laser scanning microscope as described above, in order to evaluate the transfection efficacy.

\subsection{Measurement of Fluorescence Intensities After APIM-Peptide or Leptomycin B Treatment}

HEK293 cells were transfected with pGFP-SHPRH, pGFP-SHPRH F1632A, pYFP-HLTF, or pYFP-HLTF F960A, using X-treme GENE HP transfection reagent according to manufacturer protocol (Roche diagnostics, Basel Switzerland). After 24 and $30 \mathrm{~h}$, the cells were treated with $8 \mu \mathrm{M}$ of APIM-peptide (Innovagen, Lund, Sweden), $8 \mu \mathrm{M}$ of APIM-peptide (Innovagen), or $30 \mathrm{ng} / \mathrm{mL}$ of Leptomycin B. Not treated is the negative control. Forty-five minutes after the treatment, images were taken, and the fluorescence intensity (mean) in the region of interest, covering at least 200 pixels, using Zeiss LSM 510 software, were measured.

\subsection{Preparation of Cross-Linked Cell Extracts}

HEK293T cells were transfected with the construct of interest and treated with $50 \mu \mathrm{M}$ of methyl methanesulfonate (MMS) the next day. Forty-eight hours after MMS treatment, intact cells were gently cross-linked with $0.2 \%$ formaldehyde, as previously described [24]. Cells were lysed by using $3 \times$ PCV M-PER Mammalian Protein Extraction Reagent (Thermo Scientific, Waltham, MA, USA), PIC2 $(10 \mu \mathrm{L} / \mathrm{mL}$ buffer), and PIC3 (10 $\mu \mathrm{L} / \mathrm{mL}$ buffer) (Sigma Aldrich), and complete protease inhibitor ( $20 \mu \mathrm{L} / \mathrm{mL}$ buffer) (Roche) for $1 \mathrm{~h}$ at $4{ }^{\circ} \mathrm{C}$ and sonicated in a sonication water bath 5 rounds of $30 \mathrm{~s}$ on $30 \mathrm{~s}$ off (Picoruptor SA, Diagenode). Then, $1 \mu \mathrm{L}$ of Omnicleave was added to $100 \mu \mathrm{L}$ PCV. The lysate was cleared by centrifugation for $10 \mathrm{~min}$, at $16000 \times \mathrm{g}, 4^{\circ} \mathrm{C}$.

\subsection{Immunoprecipitation}

An in-house affinity-purified rabbit polyclonal antibody raised against GFP protein, which also recognizes YFP and CFP proteins (called $\alpha$-GFP), was covalently linked to protein A paramagnetic beads (Invitrogen), according to instructions provided by New England Biolabs, Inc. Then, $750 \mu \mathrm{g}$ of cell extract was incubated with $30 \mu \mathrm{L}$ antibody-coupled beads and $0.3 \mathrm{~mL}$ of IP buffer ( $20 \mathrm{mM}$ Hepes, pH 7.9, $1.5 \mathrm{mM} \mathrm{MgCl} 2,100 \mathrm{mM} \mathrm{KCl}, 0.2 \mathrm{mM}$ EDTA, 10\% glycerol, $1 \mathrm{mM}$ DTT, $10 \mathrm{mM}$ Na-butyrate, $0.1 \mathrm{mM} \mathrm{NaVO} 3$, and $1 \mathrm{x}$ complete protease inhibitor) under constant rotation, at $4{ }^{\circ} \mathrm{C}$, overnight. The beads were washed three times with $500 \mu \mathrm{L}$ of $10 \mathrm{mM}$ Tris- $\mathrm{HCl} \mathrm{pH} 7.4$, with a 5 min incubation on ice in between each wash. The beads were resuspended in $30 \mu \mathrm{L}$ of NuPAGE (Invitrogen) loading 
buffer, including DTT (final concentration $0.1 \mathrm{M}$ ), and heated for $30 \mathrm{~min}$ at $95^{\circ} \mathrm{C}$, to reverse crosslinks before the samples were run on $4-12 \%$ Bis-Tris-HCl (NuPAGE) gels. Then, $100 \mu \mathrm{g}$ cell extract was used for input. The primary antibodies $\alpha$-GFP (ab290, Abcam) and $\alpha$-PCNA (sc-56, Santa Cruz Biotechnology), as well as the secondary antibodies IRDye 800CW (Goat Anti-Rabbit) and IRDye 700RD (Goat Anti-Mouse) (LI-COR Bioscience), were diluted in 5\% dry milk in PBS, and the proteins were visualized and quantified by using the Odyssey Imager.

Supplementary Materials: Supplementary materials can be found at http://www.mdpi.com/1422-0067/21/3/693/s1.

Author Contributions: M.O. and M.S. planned the study; M.S. performed the experiments; M.O. supervised; and M.O. and M.S. wrote the manuscript. All authors have read and agreed to the published version of the manuscript.

Funding: This work was supported by grants from Norwegian University of Science and Technology (NTNU), Trondheim, Norway).

Acknowledgments: The microscopy and MS analysis were done at the Cellular and Molecular Imaging Core Facility (CMIC), funded by the Faculty of Medicine at NTNU and Central Norway Regional Health Authority.

Conflicts of Interest: The authors declare no conflicts of interest. The funders had no role in the design of the study; in the collection, analyses, or interpretation of data; in the writing of the manuscript; or in the decision to publish the results.

\section{Abbreviations}

$\begin{array}{ll}\text { APIMPCNA } & \text { AlkB homolog 2 PCNA interacting motifProliferating cell nuclear antigen } \\ \text { CPD } & \text { Cyclo-pyrimidine dimer } \\ \text { DDT } & \text { DNA damage tolerance } \\ \text { HLTF } & \text { Helicase-like transcription factor } \\ \text { MMS } & \text { Methyl methanesulfonate } \\ \text { NER } & \text { Nucleotide excision repair } \\ \text { SHPRH } & \text { SNF2 histone-linker PHD and RING finger domain-containing helicase } \\ \text { TLS } & \text { Translesion synthesis } \\ \text { TS } & \text { Template switch } \\ \text { XPV } & \text { Xeroderma Pigmentosum Variant } \\ \text { 6-4PPs } & \text { (6-4)photoproducts }\end{array}$

\section{References}

1. Marians, K.J. Lesion Bypass and the Reactivation of Stalled Replication Forks. Annu. Rev. Biochem. 2018, 87, 217-238. [CrossRef] [PubMed]

2. Hoeijmakers, J.H. DNA damage, aging, and cancer. N. Engl. J. Med. 2009, 361, 1475-1485. [CrossRef]

3. Yoon, J.H.; Lee, C.S.; O'Connor, T.R.; Yasui, A.; Pfeifer, G.P. The DNA damage spectrum produced by simulated sunlight. J. Mol. Biol. 2000, 299, 681-693. [CrossRef] [PubMed]

4. Pfeifer, G.P. Formation and processing of UV photoproducts: Effects of DNA sequence and chromatin environment. Photochem. Photobiol. 1997, 65, 270-283. [CrossRef] [PubMed]

5. Kemp, M.G.; Sancar, A. DNA excision repair: Where do all the dimers go? Cell Cycle 2012, 11, $2997-3002$. [CrossRef]

6. Hu, J.; Adar, S.; Selby, C.P.; Lieb, J.D.; Sancar, A. Genome-wide analysis of human global and transcription-coupled excision repair of UV damage at single-nucleotide resolution. Genes Dev. 2015, 29, 948-960. [CrossRef]

7. Riou, L.; Zeng, L.; Chevallier-Lagente, O.; Stary, A.; Nikaido, O.; Taieb, A.; Weeda, G.; Mezzina, M.; Sarasin, A. The relative expression of mutated XPB genes results in xeroderma pigmentosum/Cockayne's syndrome or trichothiodystrophy cellular phenotypes. Hum. Mol. Genet. 1999, 8, 1125-1133. [CrossRef]

8. Garinis, G.A.; Mitchell, J.R.; Moorhouse, M.J.; Hanada, K.; de Waard, H.; Vandeputte, D.; Jans, J.; Brand, K.; Smid, M.; van der Spek, P.J.; et al. Transcriptome analysis reveals cyclobutane pyrimidine dimers as a major source of UV-induced DNA breaks. EMBO J. 2005, 24, 3952-3962. [CrossRef] 
9. Cordonnier, A.M.; Lehmann, A.R.; Fuchs, R.P. Impaired translesion synthesis in xeroderma pigmentosum variant extracts. Mol. Cell. Biol. 1999, 19, 2206-2211. [CrossRef]

10. Inui, H.; Oh, K.S.; Nadem, C.; Ueda, T.; Khan, S.G.; Metin, A.; Gozukara, E.; Emmert, S.; Slor, H.; Busch, D.B.; et al. Xeroderma pigmentosum-variant patients from America, Europe, and Asia. J. Investig. Derm. 2008, 128, 2055-2068. [CrossRef]

11. Kraemer, K.H.; Lee, M.M.; Andrews, A.D.; Lambert, W.C. The role of sunlight and DNA repair in melanoma and nonmelanoma skin cancer. The xeroderma pigmentosum paradigm. Arch. Derm. 1994, 130, 1018-1021. [CrossRef]

12. Kraemer, K.H.; Lee, M.M.; Scotto, J. Xeroderma pigmentosum. Cutaneous, ocular, and neurologic abnormalities in 830 published cases. Arch. Derm. 1987, 123, 241-250. [CrossRef] [PubMed]

13. Menck, C.F.; Munford, V. DNA repair diseases: What do they tell us about cancer and aging? Genet. Mol. Biol. 2014, 37, 220-233. [CrossRef] [PubMed]

14. Leung, W.; Baxley, R.M.; Moldovan, G.L.; Bielinsky, A.K. Mechanisms of DNA Damage Tolerance: Post-Translational Regulation of PCNA. Genes 2018, 10. [CrossRef] [PubMed]

15. Masuda, Y.; Suzuki, M.; Kawai, H.; Hishiki, A.; Hashimoto, H.; Masutani, C.; Hishida, T.; Suzuki, F.; Kamiya, K. En bloc transfer of polyubiquitin chains to PCNA in vitro is mediated by two different human E2-E3 pairs. Nucleic Acids Res. 2012, 40, 10394-10407. [CrossRef] [PubMed]

16. Chavez, D.A.; Greer, B.H.; Eichman, B.F. The HIRAN domain of helicase-like transcription factor positions the DNA translocase motor to drive efficient DNA fork regression. J. Biol. Chem. 2018, 293, 8484-8494. [CrossRef]

17. Ciccia, A.; Nimonkar, A.V.; Hu, Y.; Hajdu, I.; Achar, Y.J.; Izhar, L.; Petit, S.A.; Adamson, B.; Yoon, J.C.; Kowalczykowski, S.C.; et al. Polyubiquitinated PCNA recruits the ZRANB3 translocase to maintain genomic integrity after replication stress. Mol. Cell 2012, 47, 396-409. [CrossRef]

18. Betous, R.; Couch, F.B.; Mason, A.C.; Eichman, B.F.; Manosas, M.; Cortez, D. Substrate-selective repair and restart of replication forks by DNA translocases. Cell Rep. 2013, 3, 1958-1969. [CrossRef]

19. Lin, J.R.; Zeman, M.K.; Chen, J.Y.; Yee, M.C.; Cimprich, K.A. SHPRH and HLTF act in a damage-specific manner to coordinate different forms of postreplication repair and prevent mutagenesis. Mol. Cell 2011, 42, 237-249. [CrossRef]

20. Kim, J.J.; Chung, S.W.; Kim, J.H.; Kim, J.W.; Oh, J.S.; Kim, S.; Song, S.Y.; Park, J.; Kim, D.H. Promoter methylation of helicase-like transcription factor is associated with the early stages of gastric cancer with family history. Ann. Oncol. 2006, 17, 657-662. [CrossRef]

21. Capouillez, A.; Noel, J.C.; Arafa, M.; Arcolia, V.; Mouallif, M.; Guenin, S.; Delvenne, P.; Belayew, A.; Saussez, S. Expression of the helicase-like transcription factor and its variants during carcinogenesis of the uterine cervix: Implications for tumour progression. Histopathology 2011, 58, 984-988. [CrossRef] [PubMed]

22. Zhang, M.; Huang, N.; Yang, X.; Luo, J.; Yan, S.; Xiao, F.; Chen, W.; Gao, X.; Zhao, K.; Zhou, H.; et al. A novel protein encoded by the circular form of the SHPRH gene suppresses glioma tumorigenesis. Oncogene 2018, 37, 1805. [CrossRef] [PubMed]

23. Moinova, H.R.; Chen, W.D.; Shen, L.; Smiraglia, D.; Olechnowicz, J.; Ravi, L.; Kasturi, L.; Myeroff, L.; Plass, C.; Parsons, R.; et al. HLTF gene silencing in human colon cancer. Proc. Natl. Acad. Sci. USA 2002, 99, 4562-4567. [CrossRef] [PubMed]

24. Gilljam, K.M.; Feyzi, E.; Aas, P.A.; Sousa, M.M.; Muller, R.; Vagbo, C.B.; Catterall, T.C.; Liabakk, N.B.; Slupphaug, G.; Drablos, F.; et al. Identification of a novel, widespread, and functionally important PCNA-binding motif. J. Cell Biol. 2009, 186, 645-654. [CrossRef]

25. Warbrick, E. PCNA binding through a conserved motif. Bioessays 1998, 20, 195-199. [CrossRef]

26. Olaisen, C.; Kvitvang, H.F.N.; Lee, S.; Almaas, E.; Bruheim, P.; Drablos, F.; Otterlei, M. The role of PCNA as a scaffold protein in cellular signaling is functionally conserved between yeast and humans. FEBS Open Bio 2018, 8, 1135-1145. [CrossRef]

27. Muller, R.; Misund, K.; Holien, T.; Bachke, S.; Gilljam, K.M.; Vatsveen, T.K.; Ro, T.B.; Bellacchio, E.; Sundan, A.; Otterlei, M. Targeting proliferating cell nuclear antigen and its protein interactions induces apoptosis in multiple myeloma cells. PLoS ONE 2013, 8, e70430. [CrossRef]

28. Bacquin, A.; Pouvelle, C.; Siaud, N.; Perderiset, M.; Salome-Desnoulez, S.; Tellier-Lebegue, C.; Lopez, B.; Charbonnier, J.B.; Kannouche, P.L. The helicase FBH1 is tightly regulated by PCNA via CRL4(Cdt2)-mediated proteolysis in human cells. Nucleic Acids Res. 2013, 41, 6501-6513. [CrossRef] 
29. Fu, D.; Samson, L.D.; Hubscher, U.; van Loon, B. The interaction between ALKBH2 DNA repair enzyme and PCNA is direct, mediated by the hydrophobic pocket of PCNA and perturbed in naturally-occurring ALKBH2 variants. DNA Repair 2015, 35, 13-18. [CrossRef]

30. Sebesta, M.; Cooper, C.D.O.; Ariza, A.; Carnie, C.J.; Ahel, D. Structural insights into the function of ZRANB3 in replication stress response. Nat. Commun. 2017, 8, 15847. [CrossRef]

31. Choe, K.N.; Moldovan, G.L. Forging Ahead through Darkness: PCNA, Still the Principal Conductor at the Replication Fork. Mol. Cell 2017, 65, 380-392. [CrossRef] [PubMed]

32. Gederaas, O.A.; Sogaard, C.D.; Viset, T.; Bachke, S.; Bruheim, P.; Arum, C.J.; Otterlei, M. Increased Anticancer Efficacy of Intravesical Mitomycin C Therapy when Combined with a PCNA Targeting Peptide. Transl. Oncol. 2014, 7, 812-823. [CrossRef] [PubMed]

33. Sogaard, C.K.; Blindheim, A.; Rost, L.M.; Petrovic, V.; Nepal, A.; Bachke, S.; Liabakk, N.B.; Gederaas, O.A.; Viset, T.; Arum, C.J.; et al. "Two hits-one stone"; increased efficacy of cisplatin-based therapies by targeting PCNA's role in both DNA repair and cellular signaling. Oncotarget 2018, 9, 32448-32465. [CrossRef] [PubMed]

34. Sogaard, C.K.; Moestue, S.A.; Rye, M.B.; Kim, J.; Nepal, A.; Liabakk, N.B.; Bachke, S.; Bathen, T.F.; Otterlei, M.; Hill, D.K. APIM-peptide targeting PCNA improves the efficacy of docetaxel treatment in the TRAMP mouse model of prostate cancer. Oncotarget 2018, 9, 11752-11766. [CrossRef] [PubMed]

35. Fattah, F.J.; Hara, K.; Fattah, K.R.; Yang, C.; Wu, N.; Warrington, R.; Chen, D.J.; Zhou, P.; Boothman, D.A.; $\mathrm{Yu}, \mathrm{H}$. The transcription factor TFII-I promotes DNA translesion synthesis and genomic stability. PLoS Genet. 2014, 10, e1004419. [CrossRef]

36. Raeder, S.B.; Nepal, A.; Bjoras, K.O.; Seelinger, M.; Kolve, R.S.; Nedal, A.; Muller, R.; Otterlei, M. APIM-Mediated REV3L(-)PCNA Interaction Important for Error Free TLS Over UV-Induced DNA Lesions in Human Cells. Int. J. Mol. Sci. 2018, 20. [CrossRef]

37. Olaisen, C.; Muller, R.; Nedal, A.; Otterlei, M. PCNA-interacting peptides reduce Akt phosphorylation and TLR-mediated cytokine secretion suggesting a role of PCNA in cellular signaling. Cell Signal. 2015, 27, 1478-1487. [CrossRef]

38. Yoon, J.H.; Prakash, L.; Prakash, S. Highly error-free role of DNA polymerase eta in the replicative bypass of UV-induced pyrimidine dimers in mouse and human cells. Proc. Natl. Acad. Sci. USA 2009, 106, 18219-18224. [CrossRef]

39. Takaoka, K.; Kawazu, M.; Koya, J.; Yoshimi, A.; Masamoto, Y.; Maki, H.; Toya, T.; Kobayashi, T.; Nannya, Y.; Arai, S.; et al. A germline HLTF mutation in familial MDS induces DNA damage accumulation through impaired PCNA polyubiquitination. Leukemia 2019, 33, 1773-1782. [CrossRef]

40. Masuda, Y.; Mitsuyuki, S.; Kanao, R.; Hishiki, A.; Hashimoto, H.; Masutani, C. Regulation of HLTF-mediated PCNA polyubiquitination by RFC and PCNA monoubiquitination levels determines choice of damage tolerance pathway. Nucleic Acids Res. 2018, 46, 11340-11356. [CrossRef]

41. Bhat, K.P.; Cortez, D. RPA and RAD51: Fork reversal, fork protection, and genome stability. Nat. Struct. Mol. Biol. 2018, 25, 446-453. [CrossRef] [PubMed]

42. Pfeifer, G.P.; You, Y.H.; Besaratinia, A. Mutations induced by ultraviolet light. Mutat. Res. 2005, 571, $19-31$. [CrossRef] [PubMed]

43. Pfeifer, G.P.; Besaratinia, A. UV wavelength-dependent DNA damage and human non-melanoma and melanoma skin cancer. Photochem. Photobiol. Sci. 2012, 11, 90-97. [CrossRef] [PubMed]

44. Giglia-Mari, G.; Sarasin, A. TP53 mutations in human skin cancers. Hum. Mutat. 2003, 21, 217-228. [CrossRef]

45. Choi, J.H.; Pfeifer, G.P. The role of DNA polymerase eta in UV mutational spectra. DNA Repair 2005, 4, 211-220. [CrossRef]

46. Aas, P.A.; Otterlei, M.; Falnes, P.O.; Vagbo, C.B.; Skorpen, F.; Akbari, M.; Sundheim, O.; Bjoras, M.; Slupphaug, G.; Seeberg, E.; et al. Human and bacterial oxidative demethylases repair alkylation damage in both RNA and DNA. Nature 2003, 42 1,859-863. [CrossRef]

(C) 2020 by the authors. Licensee MDPI, Basel, Switzerland. This article is an open access article distributed under the terms and conditions of the Creative Commons Attribution (CC BY) license (http://creativecommons.org/licenses/by/4.0/). 